\title{
GAS DETECTORS: ACHIEVEMENTS AND TRENDS
}

\author{
Fabio Sauli
}

CERN, CH-1211 Geneva, Switzerland

\begin{abstract}
We describe recent developments of two areas of research on gaseous detectors: resistive plate chambers, aiming at best time resolution, and micropattern chambers, designed for high rate tracking in hostile environments. Emphasis is put on the progress in basic understanding of the operating features, and of the underlying physical processes. Some examples of applications of detectors outside particle physics are also provided.
\end{abstract}

Invited review talk at the $8^{\text {th }}$ Pisa Meeting on Advanced Detectors

La Biodola, Elba, Italy May 21-27, 2000

Corresponding author: fabio.sauli@cern.ch 



\section{INTRODUCTION}

Thirty years since their introduction, fast gaseous detectors, descendants of the multiwire chambers, are widely use in particle physics and other fields. With the increasingly stringent requirements of modern experiments, particularly concerning high flux operation and multi-hit capability, a large effort is devoted to the improvement of existing devices, and the development of new concepts, as apparent from the large number of contributions submitted to this conference. The following sections describe some of this works, organized under the concept of transitions.

\section{TRANSITIONS, PART ONE: SPARK TO AVALANCHE}

Parallel plate chambers (PPC) exploit avalanche multiplication in uniform electric field between two planar electrodes. Because the avalanche size depends exponentially on the distance of the primary ionization from the anode, PPC are not proportional counters. However, since an induced charge can be detected on electrodes immediately after the ionization release and the start of the avalanche, they potentially have excellent time resolution. In the spark, or Pestov counters, high detection efficiencies and good time resolution (around 50 ps) is achieved using narrow gaps, typically $100 \mu \mathrm{m}$, and gas pressures above 10 bars [1]. To avoid electronics jitters, the counters are operated in the spark mode, providing very large signals. The combined use of high resistivity electrodes (semi-conducting glass) and heavily quenched gas mixtures prevent the spread of discharges. Recent works within the ALICE collaboration have demonstrated the effectiveness of this approach to build powerful time-of-flight detectors [2].

Atmospheric pressure parallel plate counters with resistive electrodes (RPC) have been developed and in use since many years [3, 4]. Recent developments are discussed in this conference. Operated in the streamer mode, they permit to cover large areas at low cost, and are therefore suited for muon detection. While providing conveniently large signals, streamers however imply a reduced rate capability, typically limited to few hundred $\mathrm{Hz} . \mathrm{cm}^{-2}$ [5]. With the advent of cheap fast electronics, and a great amount of development work, a much better rate performance can be obtained operating the detectors in the lower gain avalanche mode. A crucial point in this respect is to reduce the probability of the transition avalanche to streamer at a given voltage. It has been found, for example, that eliminating the argon component in a freon-isobutane mixture largely increases the margin between efficient avalanche mode detection an the onset of rate-limiting streamers [6]. Similar observations have been made adding small amounts of electro-negative gases such as $\mathrm{SF}_{6}$ [7]. The typical time resolution of RPCs, $\sim 2$ ns rms at best, has been for long considered a limit for this technology [8]. More recent works however have demonstrated that time resolutions approaching those of spark counters can be achieved under suitable conditions. This is obtained with multiple gaps, electrically connected to the same amplifier [9]. In the Multi-Gap chamber (MRPC), an interesting solution is to make the intermediate resistive plates electrically floating, relying on the signal currents to build-up the appropriate potentials [10]. As an example, Fig. 1 shows the measured time resolution of a 4-gap MRPC, having 77 ps rms [11]. 
Position accuracy and timing properties of gaseous devices are limited by the low primary ionization yield; statistical dispersions are particularly large for off-normal tracks. Attempts have been made to enhance the production of secondary electrons from the cathodes, as against those released in the gas: obvious advantages are isochronism and angular independence of the signal $[12,13]$. Recent observations suggest that under certain conditions the secondary emission may be larger than previously suspected [14]. Secondary emission devices have also been developed for the detection of soft X-rays [15]. Practical use of these detectors has been however hindered by the low emission yields. A practical difficulty is to obtain a clear separation of the gas and cathode ionization components. This can be achieved measuring, in a variable gap detector, the efficiency as a function of distance between cathode and anode, and extrapolating to zero distance (some gas gap is necessary to obtain charge multiplication). Fig. 2 shows the outcome of such a measurement made with detectors having the cathodes coated with an enhanced secondary emitter (columnar grown CsI); it sets a limit of around $6 \%$ to the achievable efficiency for minimum ionizing particles [16]. Very appealing, secondary emission detectors for charged particles are certainly worth further investigations.

\section{TRANSITIONS, PART TWO: AVALANCHE TO SPARK}

Unlike the one described in the previous section, this transition is not a desirable one, and can actually lead to the destruction of the detector. MicroStrip Gas Chambers (MSGCs), introduced in 1988 [17], seemed to fulfill the most demanding requirements: high rate capability, good space accuracy, and excellent multi-track separation [18]. Quickly developed by many groups, and planned for use in major experiments planned for the new high luminosity colliders, MSGCs appeared however rather susceptible to aging and discharge damages. While the first problem could be kept at pace with a proper choice of construction materials and operating gases [19], the second appears to be an unavoidable consequence of the presence, in the field of particles to be detected, of rare but heavily ionizing tracks (nuclear fragments, gamma and neutron conversions). Fig. 3 illustrates the basic problem [20]. As a function of voltage, it provides the proportional gain, measured in laboratory conditions (low rate soft X-rays); the efficiency plateau for minimum ionizing particles is also shown, for a typical configuration and making use of currently available high-density electronics. For full efficiency, a minimum gain around 2000 has to be reached.

To simulate the effect of heavily ionizing tracks, alpha-particle emissions can be conveniently added introducing traces of ${ }^{220} \mathrm{Rn}$ in the gas flow. The ensuing discharge probability curve, measured as a function of voltage, is given in the figure; it clearly indicates the marginality of operation of the detector. Moreover, laboratory measurement may be optimistic both in rate and ionization losses as compared to realistic beam operating conditions, as found by the group developing a fast tracker for HERA-B, that led to abandon the MSGC technology for the experiment [21].

Some evidence indicates that the abnormally high gain imparted to electrons released in the high field around the edges of cathode strips could be one cause of the problem [22]. A way to delay the onset of discharges in MSGCs is the so-called advanced passivation, coating of the cathode edges 
with a thin insulating layer [23]. A recent successful test of a small but complete system of MSGCs in harsh running conditions demonstrated the validity of the approach [24]. Made with thin metallic strips on fragile supports, MSGCs remain delicate and intolerant to overloads, limiting the range of their use.

Motivated by the problems mentioned above, a large effort has been invested in finding more rugged alternatives to the MSGCs, without compromising on their performances. At the same time, detailed studies have shed new light on the transition and discharge mechanisms. The new class of devices, named Micro-Pattern Detectors (MPD), brings very promising perspectives of applications.

An innovative structure, the so-called "Compteur à Trous" (CAT) [25] consists in its basic form of a single hole in a metallic plate, facing a collecting anode. Charges released in an overlaying low field region are focussed into the hole, multiplied by the high field and detected. Surprisingly, and despite the non-uniformity and position-dependence of the field, the structure exhibits a rather good proportionality. The micro-CAT detector, a multi-hole extension of CAT, permits to cover larger areas; it is being used in a variety of medical imaging applications [26].

Introduced at about the same time, MICROMEGAS exploits the parallel plate multiplication in thin gaps between a fine mesh and an anode plate [27]. Recent basic developments and performances of large size detectors are described in a contribution to this conference [28]. Fig. 4 provides as an example the gain measured in different gases. Whilst frequently discharging in harsh beam conditions, the detectors have been demonstrated not to suffer permanent damages. At a gain around 5000, required for full efficiency, the discharge rate in the mixture $\mathrm{Ne}-\mathrm{C}_{2} \mathrm{H}_{6}-\mathrm{CF}_{4}$ is $0.1 \mathrm{~Hz}$, considered acceptable by the experiment and resulting in no permanent damages to the detector.

The Gas Electron Multiplier (GEM) is another innovative multiplying structure [29]. It consists of a set of holes, typically 50-100 $\mu \mathrm{m}$ in diameter, chemically etched through a metal-insulator-metal thin-foil composite ${ }^{1}$ (Fig. $5)$. With proper choice of potentials, electrons released in the gas above the foil are drifted into the holes, and multiplied in avalanche in the high field within the channels. Most of the electrons in the avalanche move into the lower gap and are collected; effective gains well above several thousand can be achieved, sufficient for detection of minimum ionizing tracks in thin conversion layers [30]. Localization can then be performed collecting the charge on a patterned one- or two-dimensional readout board [31]. A unique feature of GEM detectors is that proportional multiplication and charge detection are performed on separate electrodes; with proper choice of the operating conditions one can effectively avoid the propagation of discharges to the sensitive electronics.

Exploiting the kapton-etching technology developed for making GEM electrodes, other promising multiplying structures have been recently developed: the micro-groove [32], the WELL [33], the micro-wire detector [34]. All devices permit to reach gains above $10^{4}$. However, a systematic study of their behavior reveals the similarity of values for the maximum sustainable gain, several thousand, when exposed to alpha particles [20]. As an example, Fig. 6 shows the behavior for a single GEM detector.

\footnotetext{
${ }^{1}$ Technology developed at CERN-EST by A. Gandi and R. De Oliveira
} 
The appearance of discharges is attributed to the avalanche size exceeding a critical value, in the range $10^{7}-10^{8}$ (the so-called Raether limit); the ensuing field distortion induces a spontaneous, photon-mediated transition from a proportional avalanche to a streamer. In MPDs, having a rather high field over small distances, a further transition from streamer to discharge is very likely to occur.

A similar reduction in maximum gain has been observed exposing the detectors to increasing fluxes of X-rays. In this case, it is not very clear however how the total avalanche charge can reach the limit, unless one invokes the presence of long-lived excited states or surface charge accumulations followed by spontaneous emission [35].

Cascading several multiplying structures permits to increase the value of gain at discharge, as observed long ago with the multi-step chamber [36]. This can be easily achieved with the GEM, used as a pre-amplifier in combination with another MPD. This is the solution adopted for the above mentioned HERA-B experiment, and has led to the first large production of GEM electrodes: more than 200 foils, $27 \times 25 \mathrm{~cm}^{2}$ each, have been manufactured at CERN. Fig. 7 shows the gain and discharge rates measured with the double structure; comparison with Figs. 3 and 6 indicates the substantial increase of the operational margin. The MSGC-GEM detectors are at present gradually installed at DESY [37].

Similar performances are obtained with multiple GEM devices, intrinsically cheaper and more robust than MSGCs. Double and triple GEM detectors have been extensively tested in particle beams [30, 38, 39], and fourstage GEMs are under development for single photoelectron detection [40]. With a double GEM, gains in excess of $10^{4}$ can be obtained at very high rates and in presence of heavily ionizing tracks [20]. In Fig. 8, the discharge probabilities on irradiation with alpha particles are plotted as a function of gain for a double and a triple GEM. The exact reasons for the improvement are not completely clear. It is suspected that, since in cascaded system the same overall gain is obtained with each element operated at much reduced voltage, the amount of charge required for the transition is larger, or in other words that the Raether limit is voltage dependent. Alternatively, one can invoke the larger avalanche spread in multiple structures to reduce the charge density [35]. Systematic measurements of discharge limits in various gases show that the limit is higher in mixtures with smaller quencher content, having at the same time larger diffusion and lower operating voltage [41]. The physical separation between multiplying elements plays a fundamental role, as demonstrated by measurements made with the so-called Super-GEM: two foils with holes aligned and in contact provide the same performances as a single GEM [42]. Similar observations have been made by other authors [43].

The discharge probability depends on the rate and nature of the heavily ionizing background, and is therefore experiment dependent; even if small, it cannot be assumed to be zero. It is therefore necessary to ensure that no permanent damages to the structures and to the electronics result from accidental sparking. In GEM detectors, the energy of a discharge can be reduces subdividing one electrode in electrically separated segments, individually powered through high value resistors. Discharge propagation mechanisms between GEMs and from the last GEM to the readout electrode have been extensively studied $[44,45]$. Whilst the propagation between GEMs 
seems to be field-independent and very fast, suggesting photons to be the culprit, the more dangerous propagation to the anodes only appears at high transfer fields. Moreover, the full discharge probability appears to depend on the energy of the initial spark, as shown in Fig. 9 [44].

A set of large size ( $31 \times 31 \mathrm{~cm}^{2}$ active) double-GEM detectors is in construction at CERN for the needs of the COMPASS experiment; prototypes have been successfully tested in realistic experimental conditions [38].

\section{TRANSITIONS, PART THREE: FROM PARTICLE PHYSICS TO APPLICATIONS}

Detectors originally developed for particle physics have been often adapted to specific needs of other applied fields. A special section in this conference covers many recent developments. Here I will briefly mention some interesting uses and applications of the GEM technology.

In ESA's Gamma-Ray Astrophysics Laboratory (INTEGRAL), to be launched in 2001, one of the detectors is a coded aperture X-ray imager making use of a large area circular MSGC [46]. Concerns about long-term reliability of the device, exposed to sudden swarms of ionizing particles from solar wind, suggested to consider the addition of a GEM pre-amplifier to the existing structure. In order to increase the reliability, and circumscribe the loss of efficiency in case of a local damage, the circular GEMs, $25 \mathrm{~cm}$ in diameter, have been segmented in 16 individually powered sectors (Fig. 10).

Two-dimensional GEM detectors have been used to obtain X-ray absorption images, in view of possible applications for medical diagnostics. A unique feature of the devices is that a global signal can be detected on the GEM electrode facing the readout, and used for triggering and event selection. Fig. 11 shows an $8 \mathrm{keV}$ absorption radiography of a small mammal; the image size is $\sim 6 \times 3 \mathrm{~cm}^{2}$, and the position resolution (limited by the photoelectron range in the gas) around $100 \mu \mathrm{m}$ rms. The image was recorded using a high-density analogue encoder system, developed for particle physics, rather limited in the readout speed. Further use of the technology demands the development of faster dedicated read-out electronics. To avoid the reconstruction ambiguities in case of multiple events, we have developed the 3-D projective readout scheme described in previous work [31]. In the socalled hexaboard detector, the readout plane is patterned with contiguous hexagonal pads interconnected in rows along three directions ${ }^{2}$ (Fig. 12). For each event, the position of the event reconstructed unambiguously.

Multiple GEM structures permit to achieve large gains in pure noble gases [47]; with proper choice of geometry and fields, both photon and ion feedback can be strongly suppressed [48]. Recent work has demonstrated the feasibility of large area, position-sensitive gaseous photo-multipliers based on the technology [40]. The photoelectron extraction and transport properties through the multiple structure have been modeled with the help of simulation programs [49] and confirmed experimentally [50]. To obtain a good electron extraction, a high field needs to be applied between the photocathode and the first GEM in the structure. This is however in conflict with obtaining a good collection into the GEM holes, as a fraction of the field lines lead to the metal. A moderate parallel plate amplification between cathode and first GEM, helped by electron diffusion, overcomes the limitation and

\footnotetext{
${ }^{2}$ Technology developed by R. De Oliveira at CERN.
} 
results in a position-independent detection efficiency, see Fig. 13 [49].

In applications were fast electronics detection of individual events is not required, an optical imaging system has considerable advantages in terms of simplicity of the set-up [51]. A GEM detector with a semi-transparent anode is observed by an image-intensified CCD camera, recording the light flashes produced by gas scintillation in the avalanches. The approach, that takes full advantage of existing commercial image acquisition and handling systems, is certainly very promising. An example of absorption radiography made with the imager is given in Fig. 14.

\section{REFERENCES}

[1] Y. Peskov, Nucl. Instrum. Methods 196 (1982) 45.

[2] H.R. Schmidt, Nucl. Phys. B (Proc. Suppl.) 78 (1999) 372.

[3] R. Santonico, R. Cardarelli, Nucl. Instrum. Methods 187 (1981) 377.

[4] R. Cardarelli, R. Santonico, Nucl. Instrum. Methods A 263 (1988) 200.

[5] Y. Arai et al, Nucl. Instrum. Methods 367 (1995) 398.

[6] V.V. Ammosov et al, Nucl. Instrum. Methods A 441 (2000) 348.

[7] P. Camarri et al, Nucl. Instrum. Methods A 414 (1998) 317.

[8] M. Abbrescia et al, Nucl. Instrum. Methods A 431 (1999) 413.

[9] P. Fonte et al, Nucl. Instrum. Methods A 443 (2000) 201.

[10] E. Cerron-Zeballos et al, Nucl. Instrum. Methods A 374 (1996) 132.

[11] ALICE Technical Design Report, CERN/LHCC 2000-12 (2000).

[12] R. Chechik et al, Nucl. Instrum. Methods A 342 (1994) 458.

[13] E. Ceron Zeballos et al, Nucl. Instrum. Methods A 392 (1997) 150.

[14] P. Fonte, V. Peskov, High-resolution TOF with RPC. 5th Int. Conf. on Position-Sensitive Detectors (London, 1999). Subm. Nucl. Instrum. Methods.

[15] A. Breskin et al, Nucl. Instrum. Methods A 348 (1994) 207.

[16] I.J. Park et al, IEEE Trans. Nucl. Sci. NS-46 (1999) 275.

[17] A. Oed, Nucl. Instrum. Methods A 263 (1988) 351.

[18] F. Sauli, Nucl. Phys. 61 B (1998) 236.

[19] J. Bohm et al Nucl. Instrum. Methods A360 (1995) 34.

[20] A. Bressan et al., Nucl. Instrum. Methods A424 (1998) 321.

[21] B. Schmidt, Nucl. Instrum. Methods A 419 (1998) 230.

[22] R. Bouclier et al., Nucl. Instrum. Methods A365 (1995) 65.

[23] R. Bellazzini et al, Nucl. Instrum. Methods A398 (1998) 426.

[24] R. Bellazzini et al., The CMS Micro-Strip Gas Chamber project, Subm. Nucl. Instrum. Methods (2000).

[25] F. Bartol et al, J. Phys. III France 6 (1996) 337.

[26] A. Sarvestani et al, Nucl. Instrum. Methods A 419 (1998) 444.

[27] I. Giomataris et al, Nucl. Instrum. Methods A376 (1996) 29.

[28] P. Abbon et al., Micromegas as a fast gaseous microstrip detector for the COMPASS experiment, 8th Pisa Meeting on Advanced Detectors (Isola d'Elba, Italy, 21-27 May 2000). Subm. Nucl. Instrum. Methods (2000).

[29] F. Sauli, Nucl. Instrum. Methods A386 (1997) 531.

[30] J. Benlloch et al, Nucl. Instrum. Methods A 419 (1998) 410.

[31] A. Bressan et al, Nucl. Instrum. Methods A425 (1999) 254.

[32] R. Bellazzini et al., Nucl. Instrum. Methods A 424 (1998) 444.

[33] R. Bellazzini et al., Nucl. Instrum. Methods A 423 (1998) 125.

[34] B. Adeva et al, Nucl. Instrum. Methods A 435 (1999) 402.

[35] V. Peskov et al, IEEE Trans. Nucl. Sci. NS-45 (1998) 244. 
[36] G. Charpak, F. Sauli, Phys. Letters 78 B (1978) 523.

[37] T. Zeuner, Nucl. Instrum. Methods A 446 (2000) 324.

[38] S. Bachmann et al, CERN-EP/99-148. Subm. Nucl. Instrum. Methods (1999).

[39] M. Ziegler et al, A triple GEM detector for LHCb, TRAC 99-024 (1999).

[40] A. Buzulutskov et al, Nucl. Instrum. Methods A 443 (2000) 164.

[41] Unpublished measurements of the author (1999).

[42] C. Büttner et al., Nucl. Instrum. Methods A 409 (1998) 79.

[43] R. Bellazzini et al, Nucl. Instr. Methods A425 (1999) 218.

[44] S. Bachmann et al, Discharge mechanisms in GEM detectors, in preparation.

[45] M. Wallmark, Operating limits of a Gas Electron Multiplier for Portal Imaging. Thesis at Karolinska Institute Karolinska Institute (2000).

[46] N. Lund et al, Astrophysical Lett. and Communications 39 (1999) 339.

[47] A. Bressan et al, Nucl. Instrum. Methods A 423 (1998) 119.

[48] G. Garty et al, Nucl. Instrum. Methods A 433 (1999) 476.

[49] A. Sharma, CERN-OPEN-99/372. Subm. Nucl. Instrum. Methods.

[50] C. Richter et al, On the efficient electron transfer through GEM. Subm. Nucl. Instrum. Methods (2000).

[51] F. Fraga et al, IEEE Trans. Nucl. Sci. NS-47(2000).

\section{FIGURE CAPTIONS}

Fig. 1: Best time resolution of a multi-gap RPC.

Fig. 2: Efficiency vs. gas gap measured in a detector with enhanced secondary cathode emission.

Fig. 3: Gain, efficiency and discharge probability as a function of voltage in a MSGC.

Fig. 4: Gain vs. voltage of MICROMEGAS in different gas mixtures.

Fig. 5: Schematics and electric field in the Gas Electron Multiplier.

Fig. 6: Gain and discharge probability on alpha particles of GEM.

Fig. 7: Gain and discharge probability in the MSGC-GEM detector.

Fig. 8: Discharge probability on alphas of the double- and triple-GEM.

Fig. 9: Discharge propagation probability to the anodes in a double-GEM detector, as a function of field, for different GEM segmentations.

Fig. 10: Large area segmented GEM developed for the INTEGRAL detector.

Fig. 11: Absorption radiography of a small mammal recorded with a 2-D GEM detector.

Fig. 12: The hexaboard: hexagonal readout pads connected in rows along three readout directions.

Fig. 13: Avalanche size for single photoelectrons, computed as a function of distance from the GEM's hole centers, under different assumptions.

Fig. 14: X-ray absorption radiography recorded with a CCD camera on a scintillating GEM detector. 


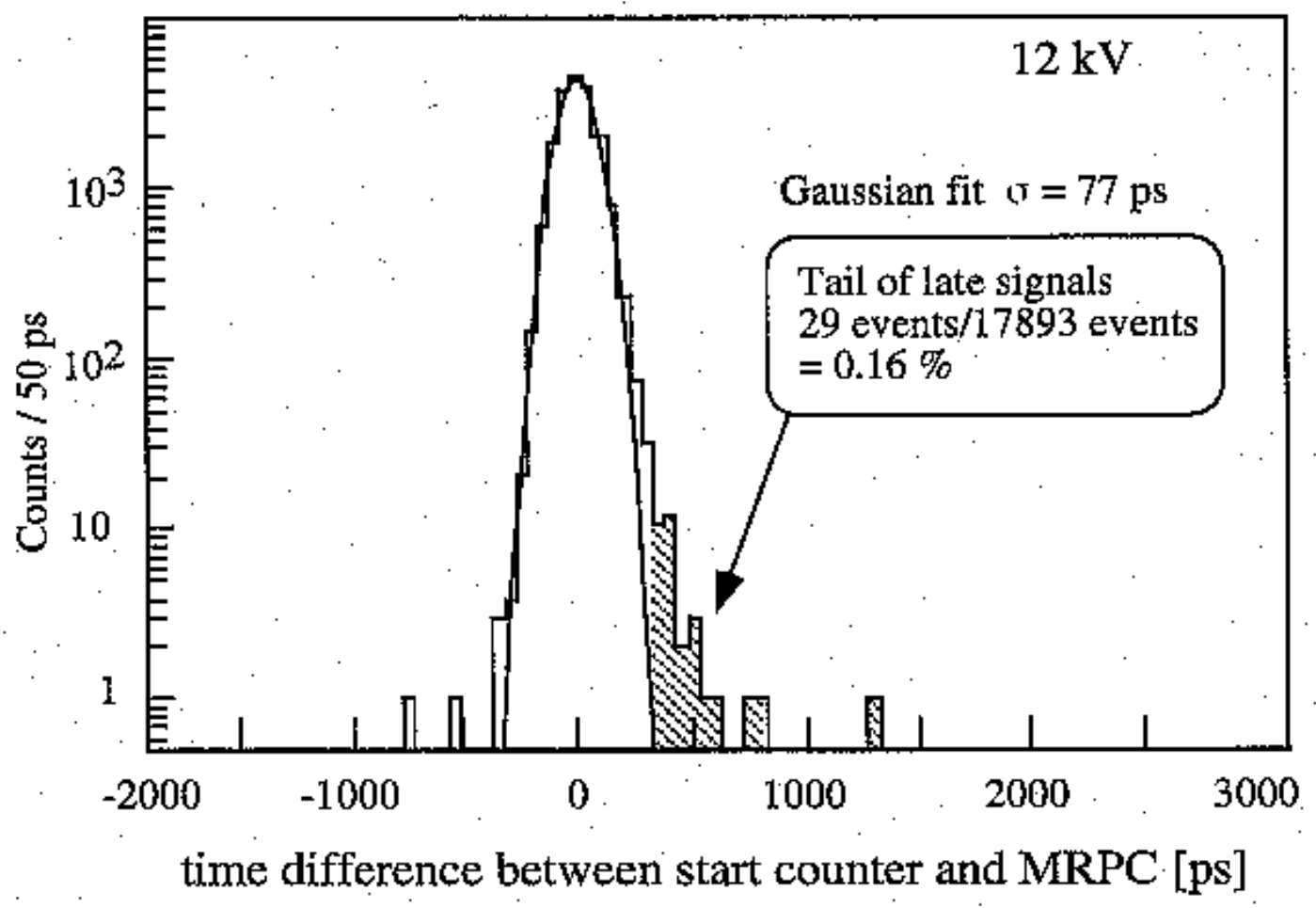

Fig. 1

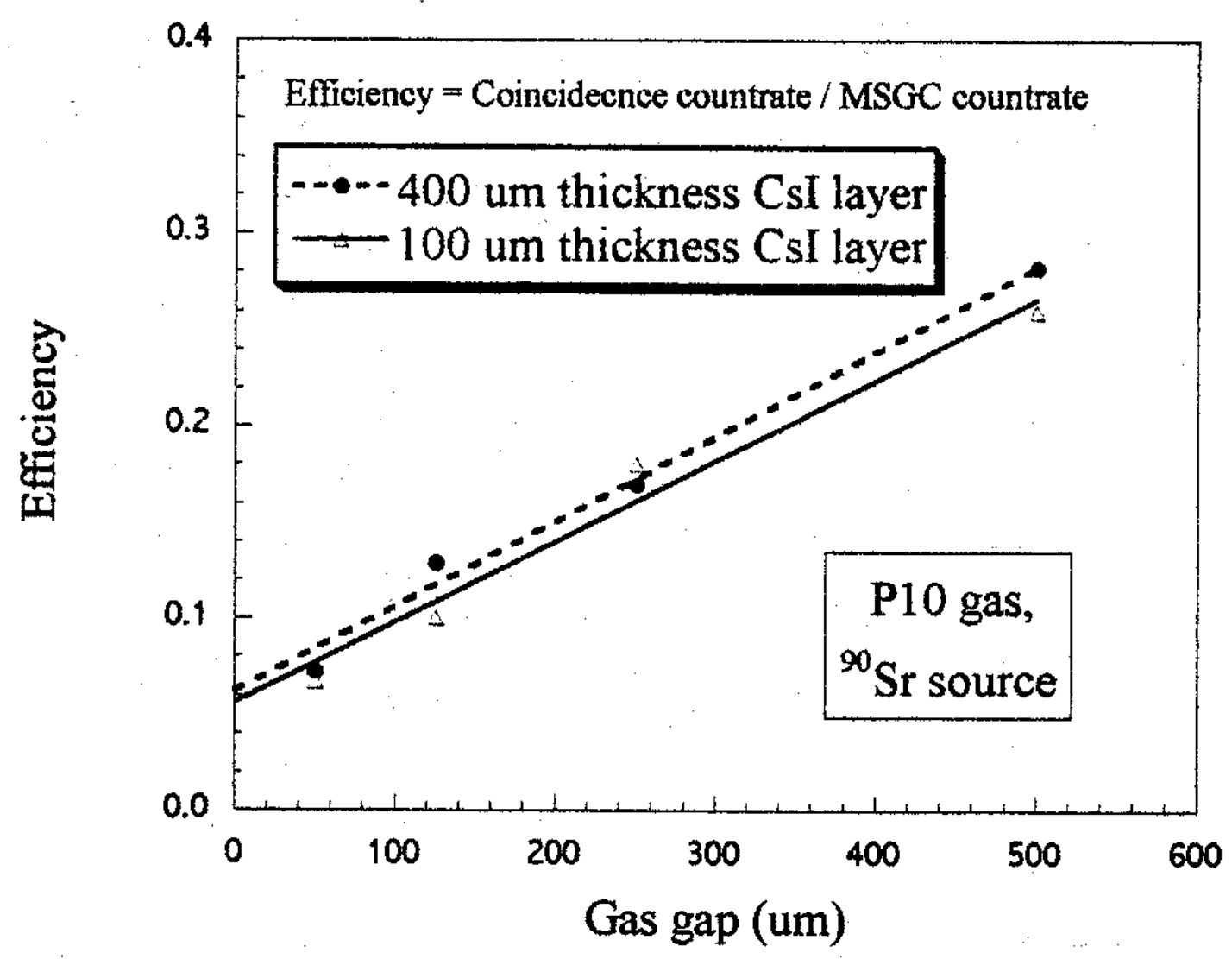

Fig. 2 


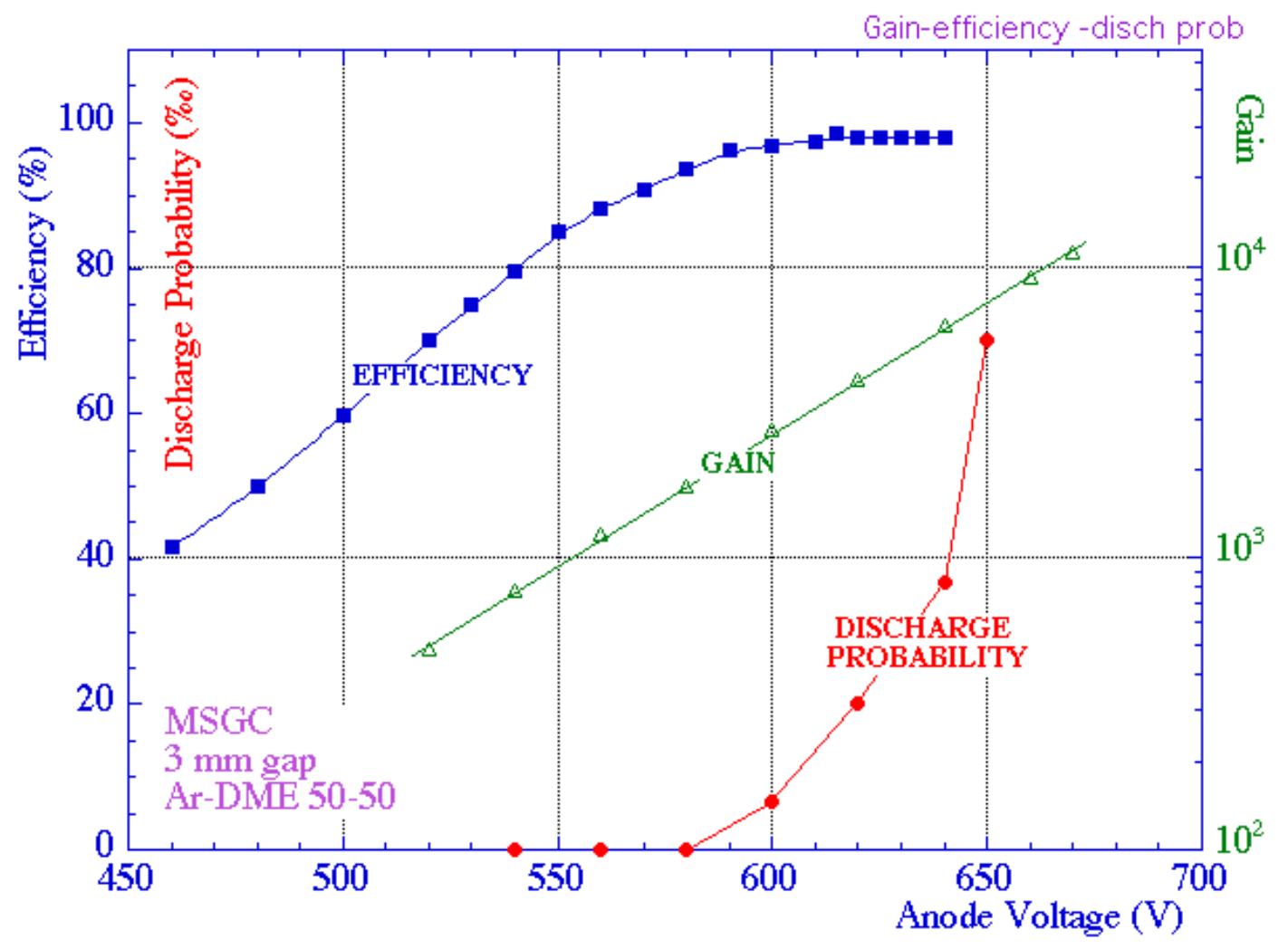

Fig. 3

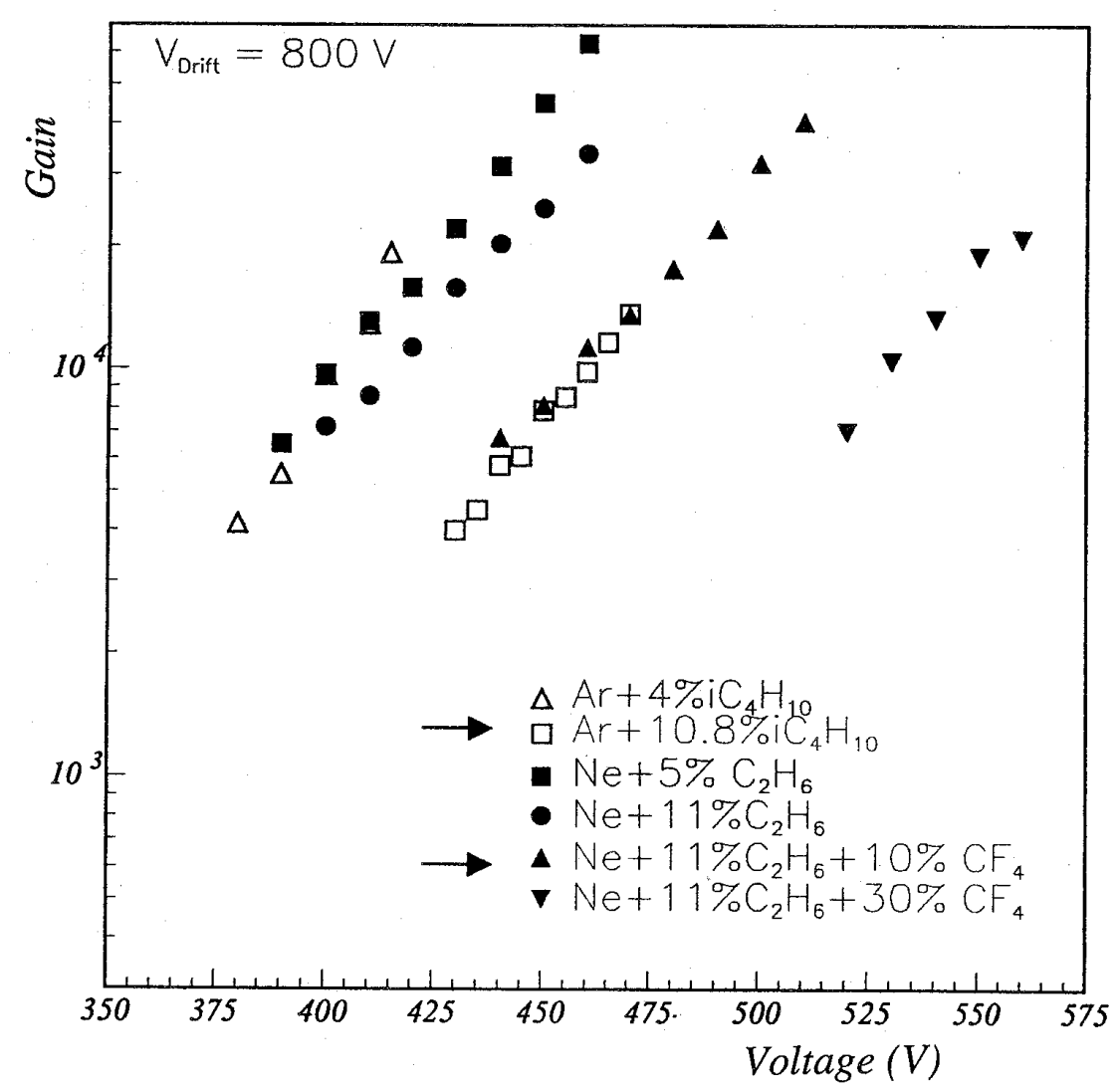

Fig. 4 


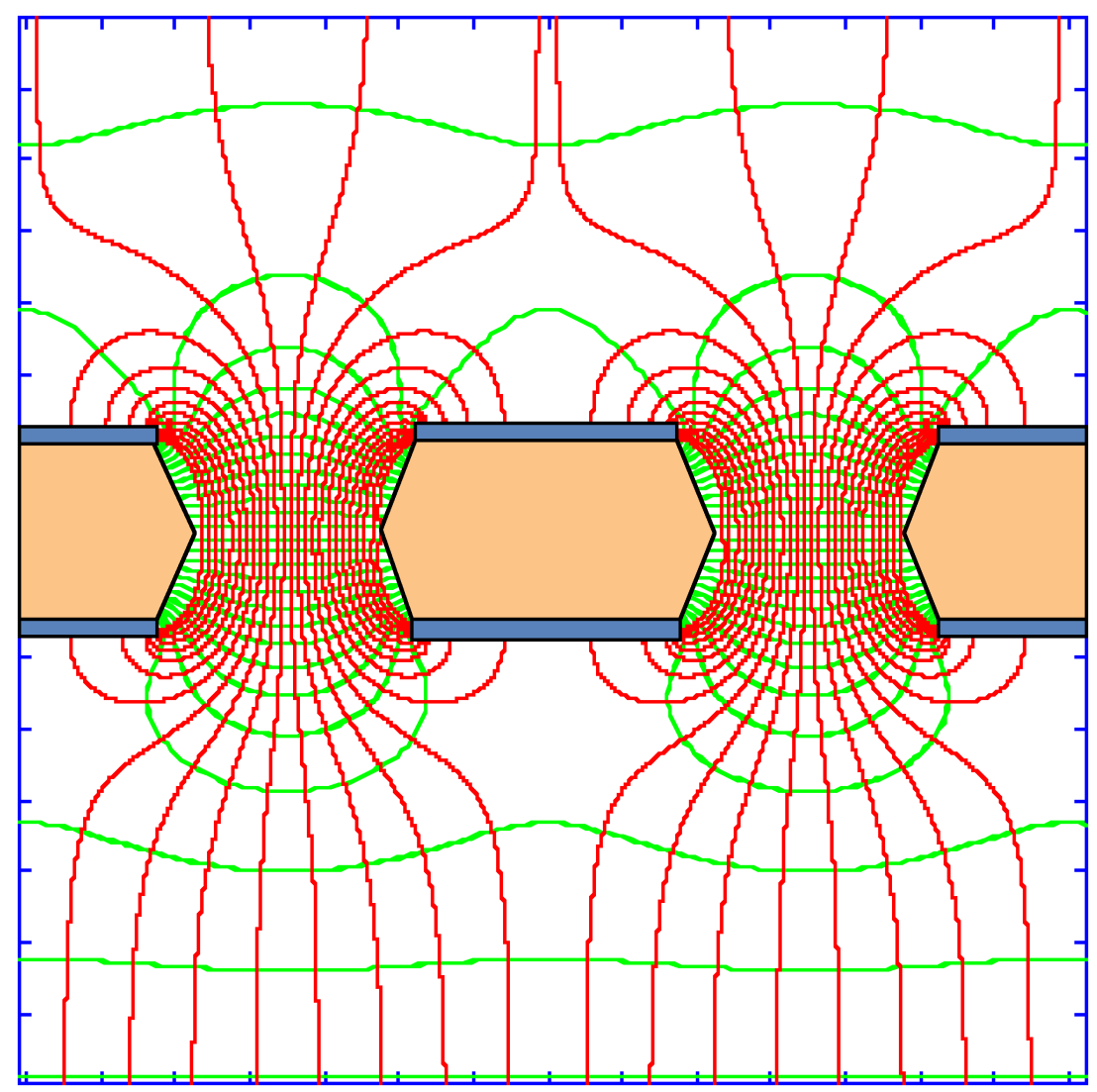

Fig. 5

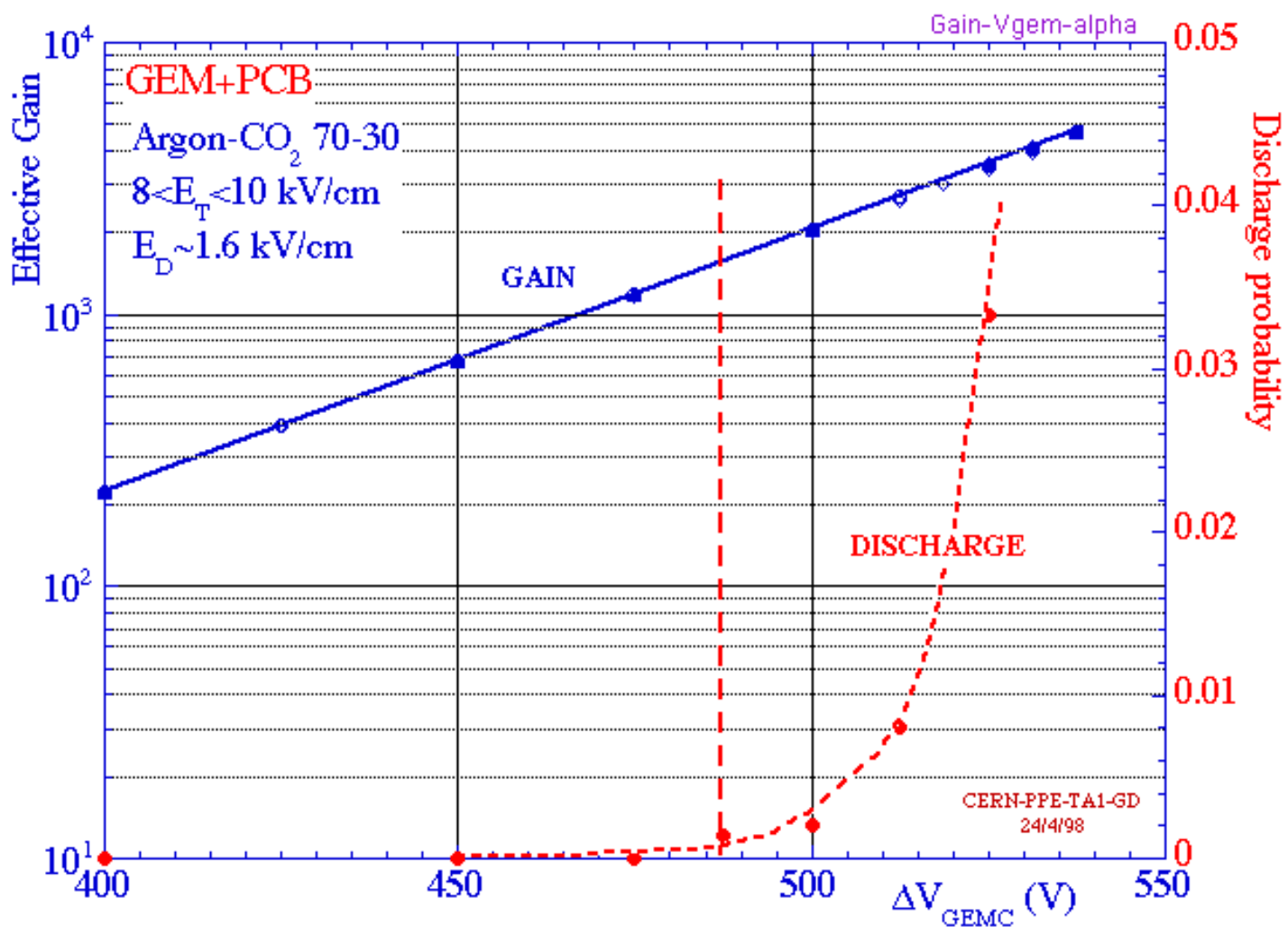

Fig. 6 


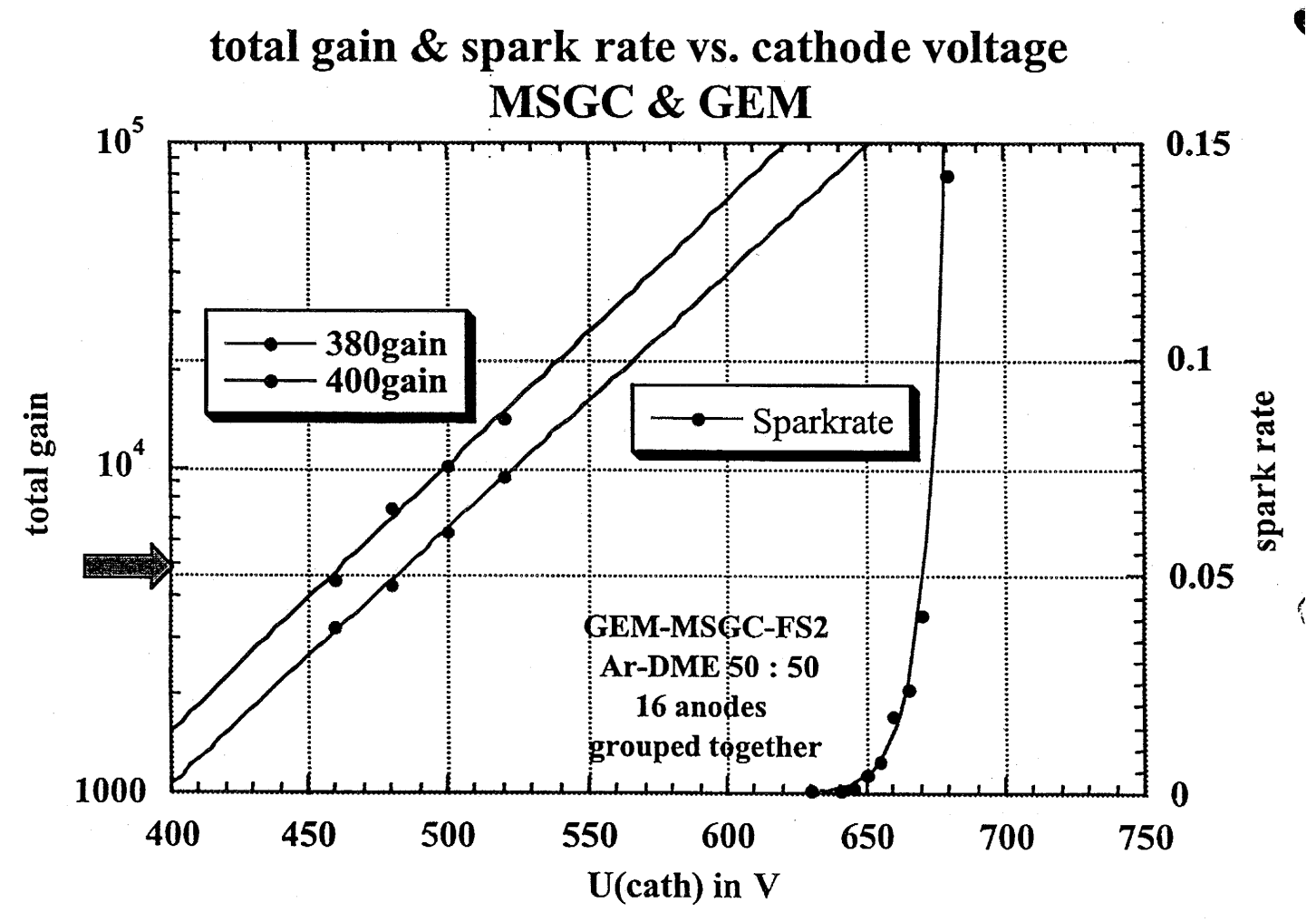

Fig. 7

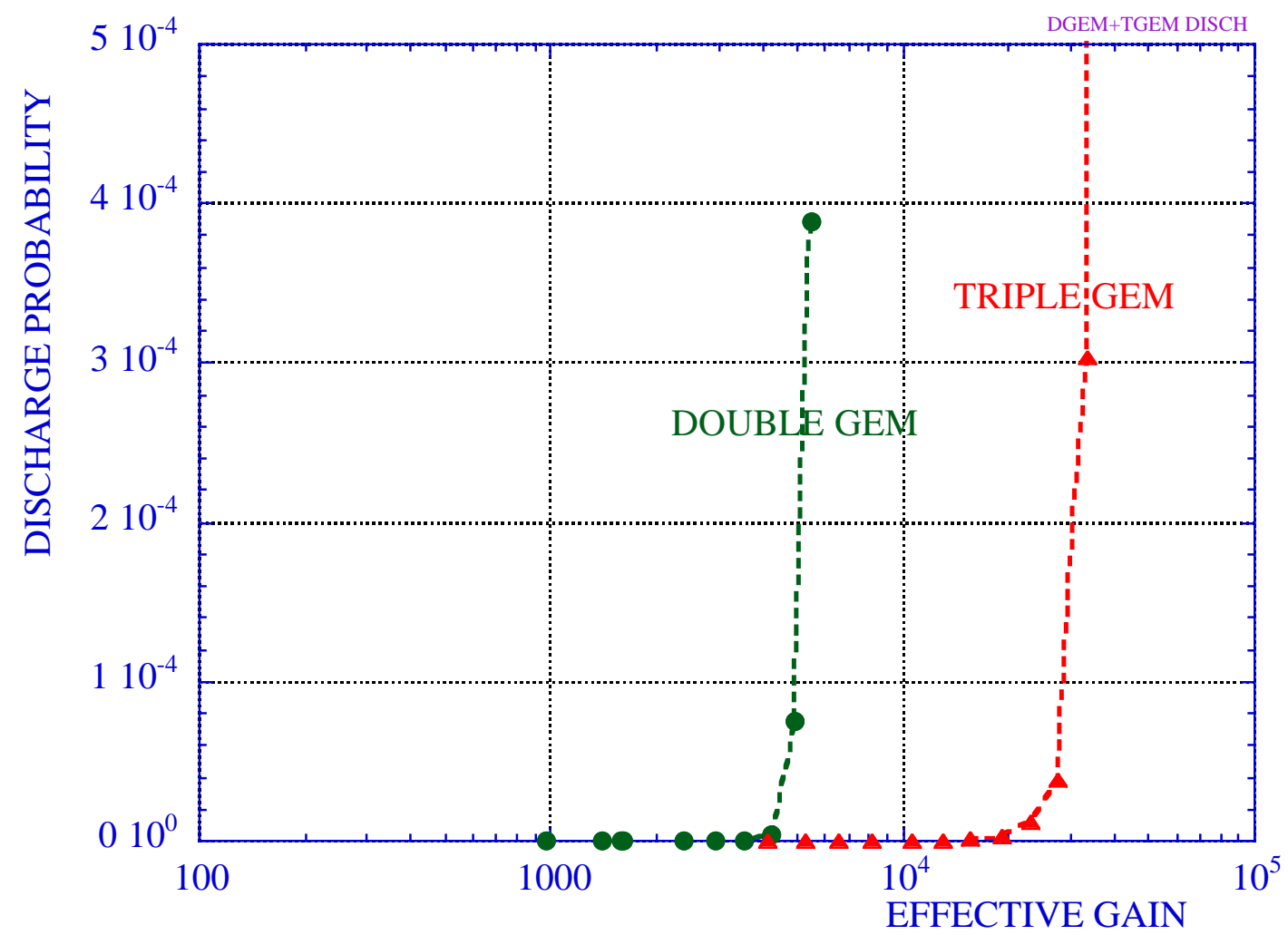

Fig. 8 


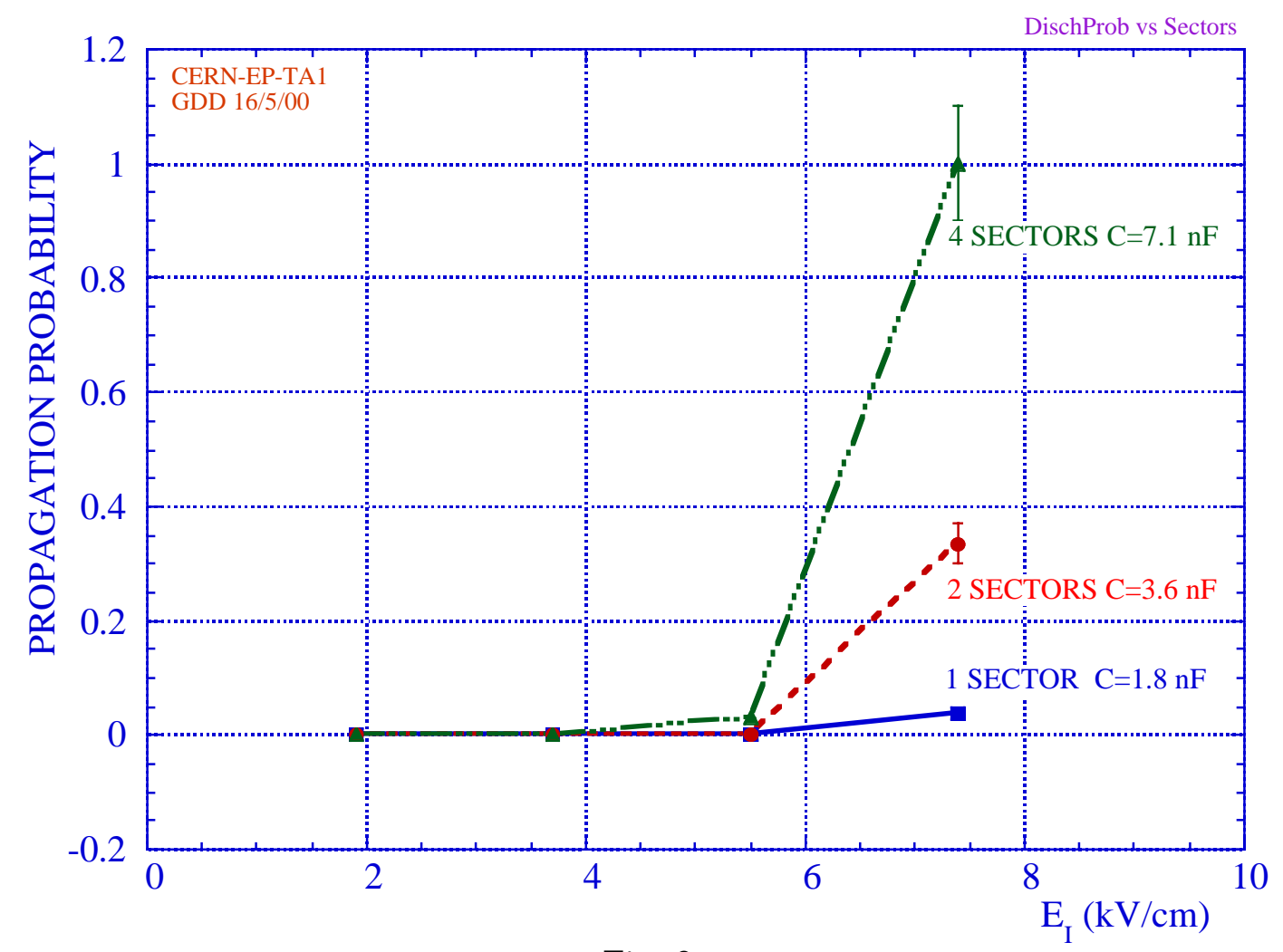

Fig. 9

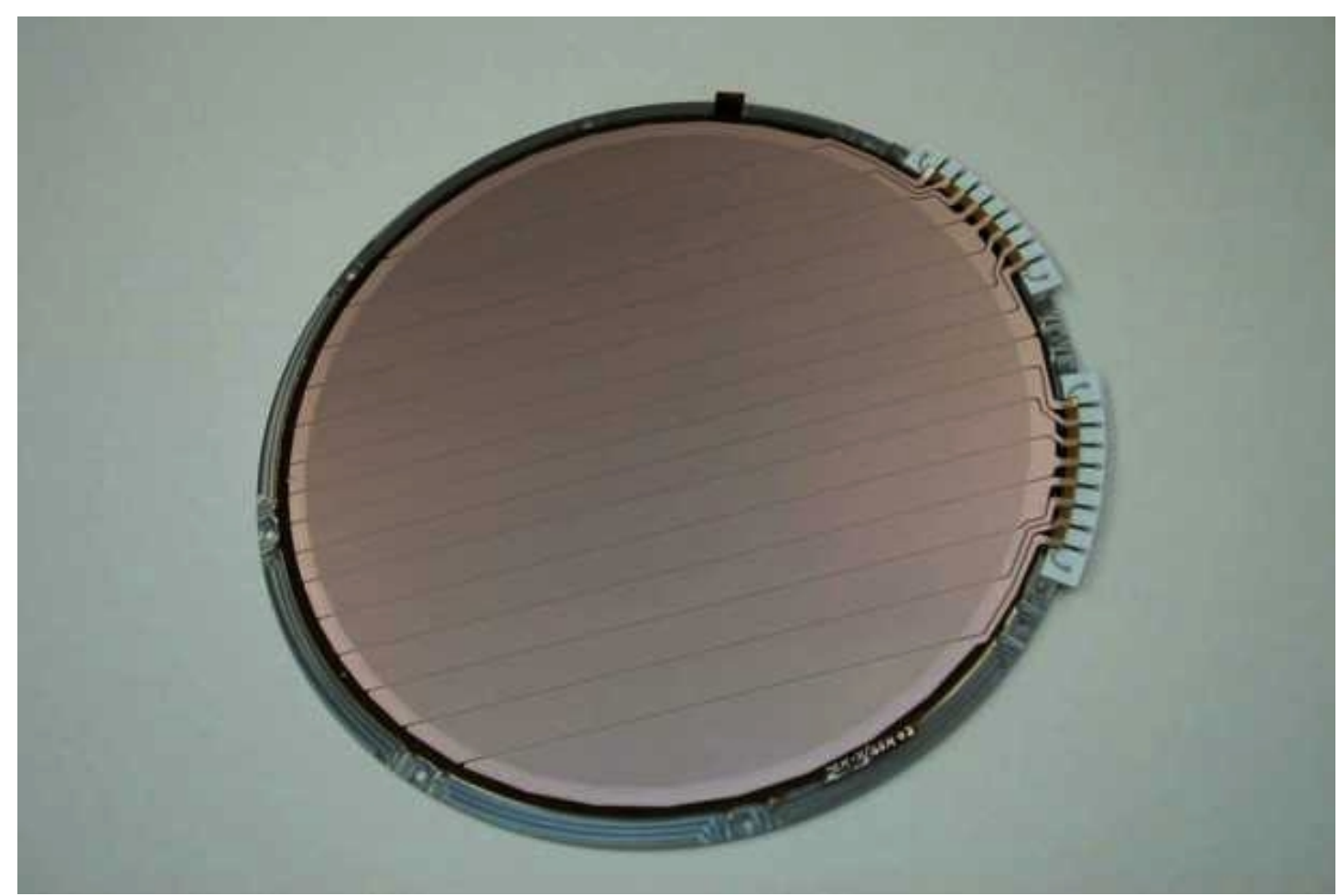

Fig. 10 


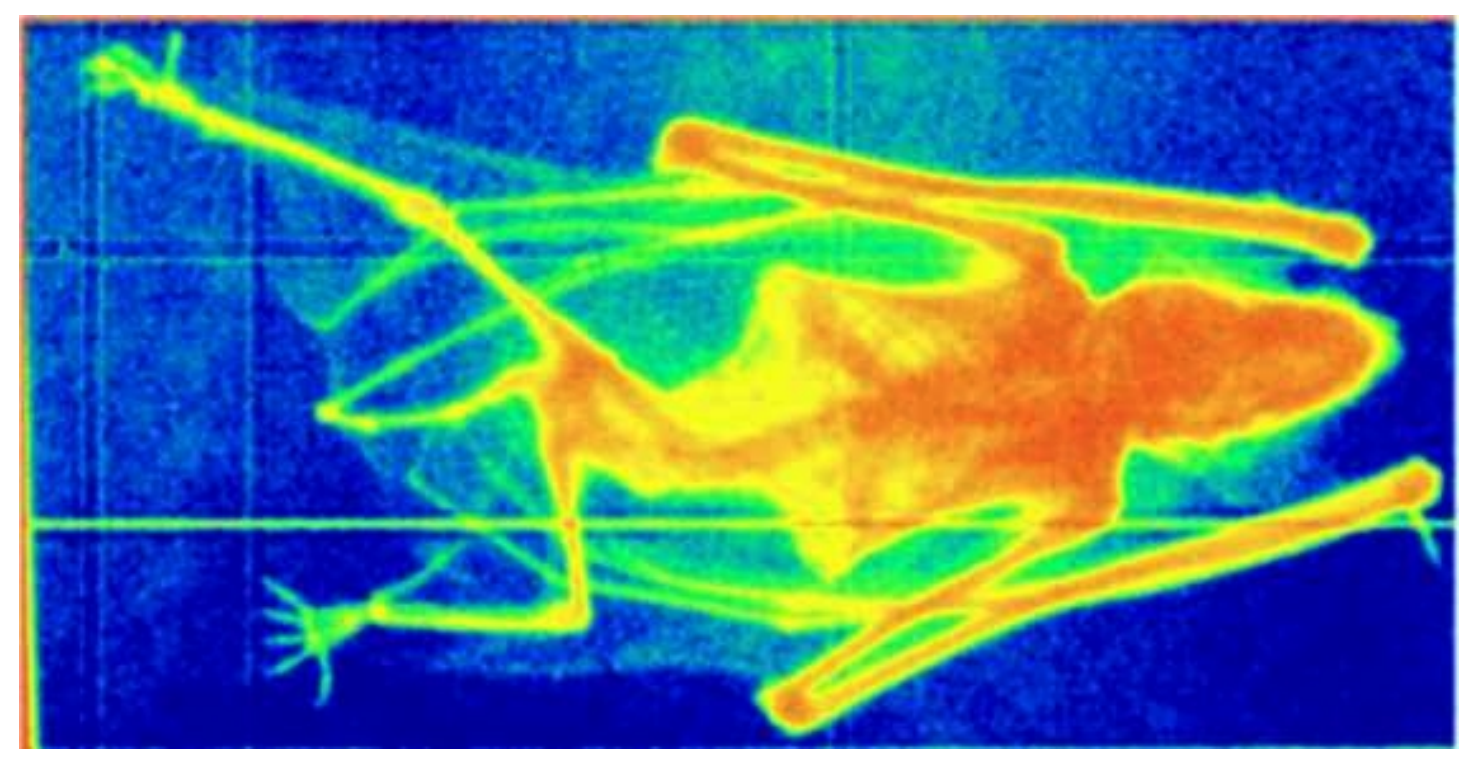

Fig. 11

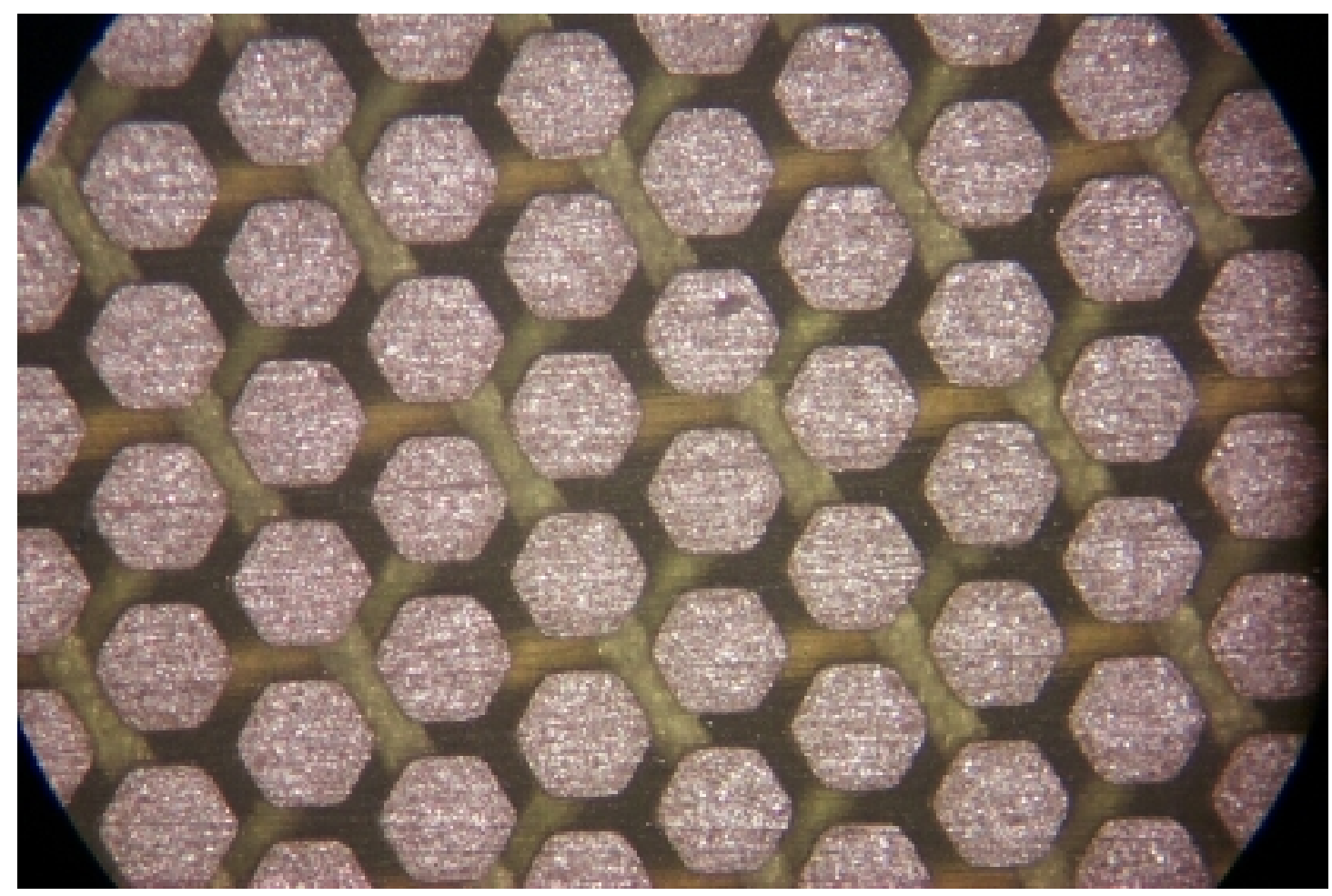

Fig. 12 


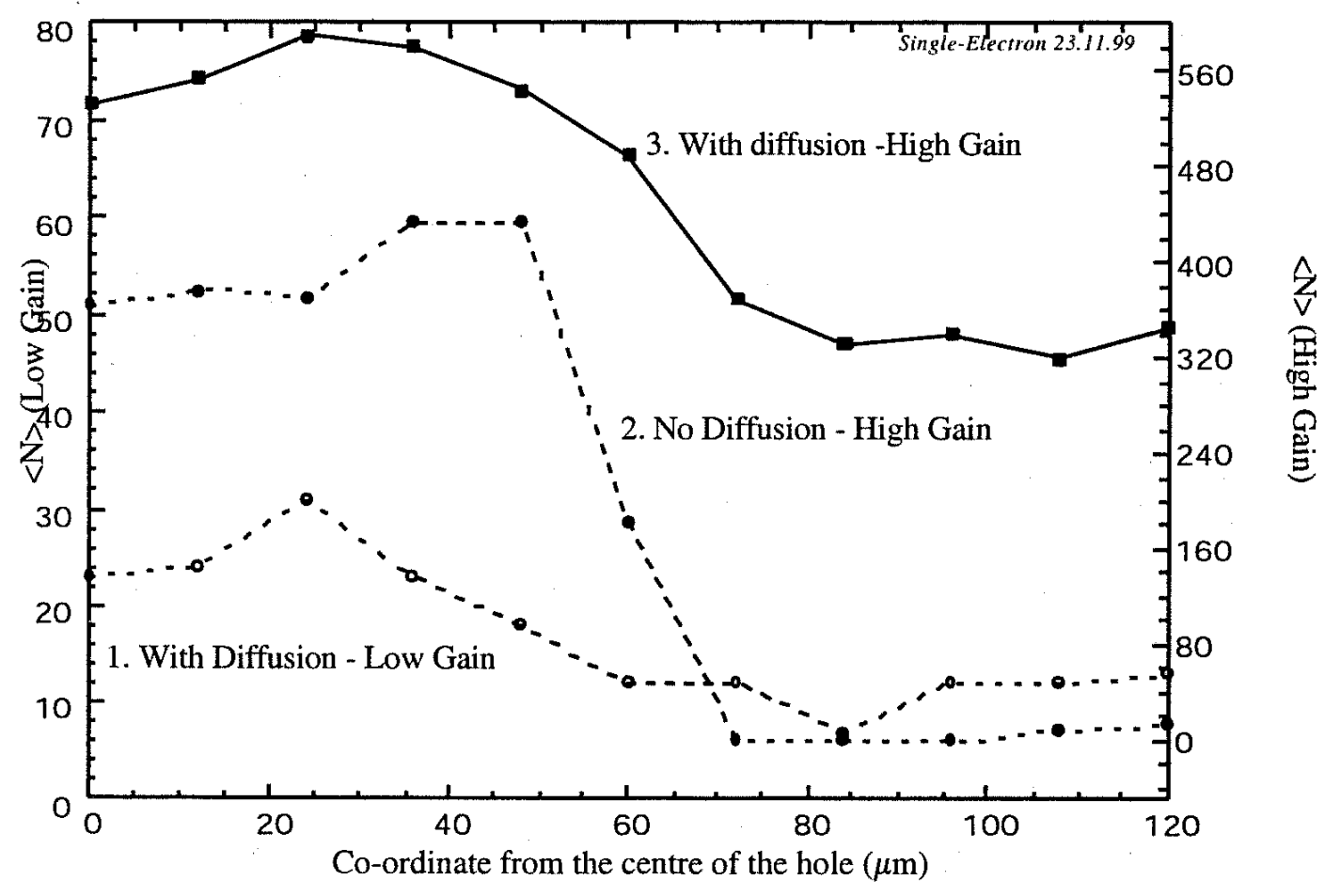

Fig. 13

Fig. 14 